\title{
Da proximidade ao distanciamento social: desafios de sustentar a lógica da atenção psicossocial em tempos de pandemia.
}

Relato de experiência do Centro de Atenção Psicossocial Infantil - CAPSi Asa Norte no Distrito Federal

\section{From proximity to social isolation: challenges to sustain the logic of psychosocial care in times of pandemic. \\ Experience report of the Child Psychosocial Care Center - CAPSi Asa Norte in the Federal District}

\author{
Danyelle Souza Martins ${ }^{1}$ \\ Jean Carlos Costa Vieira ${ }^{2}$ \\ Maírla Soares Rolim Castro ${ }^{3}$ \\ Milena de Sousa Lima ${ }^{4}$ \\ Natália Monteiro Portella ${ }^{5}$ \\ Renata Priscila Oliveira Fonseca ${ }^{6}$ \\ Sara Hussein Garcia de Figueiredo ${ }^{7}$
}

Resumo: O presente estudo descritivo busca relatar a vivência de residentes do Programa de Residência em Saúde Mental Infantojuvenil em um Centro de Atenção Psicossocial da Infância (CAPSi DF) no contexto de pandemia relacionado ao novo coronavírus (COVID -19). Considerando as orientações da Organização Mundial de Saúde, Ministério da Saúde e os planos de contingenciamento do governo local, as ações de reabilitação psicossocial em saúde mental Infantojuvenil passaram adaptações. Recorreu-se a uma metodologia qualitativa ancorada na atenção psicossocial, princípios norteadores dos CAPS, reforma psiquiátrica e na literatura sobre loucura, cultura e sociedade para promover uma reflexão crítica sobre a necessária tarefa de sustentar a lógica da atenção psicossocial em tempos de isolamento social e agravamento dos riscos à saúde mental das crianças e dos adolescentes.

Palavras-Chaves: Pandemia; Atenção Psicossocial; Loucura; Saúde Mental Infantojuvenil; Saúde Pública;

\footnotetext{
1 Terapeuta Ocupacional residente em Saúde Mental Infantojuvenil. Escola Superior de Ciências da Saúde ESCS/FEPECS/SES-DF;

2 Enfermeiro residente em Saúde Mental infantojuvenil. Escola Superior de Ciências da Saúde ESCS/FEPECS/SES-DF;

3 Assistente Social preceptora em Saúde Mental Infantojuvenil. Escola Superior de Ciências da Saúde ESCS/FEPECS/SES-DF e servidora da Secretaria de Saúde do Distrito Federal- SESDF;

${ }^{4}$ Psicóloga residente em Saúde Mental Infantojuvenil. Escola Superior de Ciências da Saúde ESCS/FEPECS/SES-DF;

${ }^{5}$ Psicóloga preceptora em saúde em saúde mental infantojuvenil. Escola Superior de Ciências da Saúde ESCS/FEPECS/SES-DF e servidora da Secretaria de Saúde do Distrito Federal- SESDF;

6 Assistente Social residente em Saúde Mental Infantojuvenil da Escola Superior de Ciências da Saúde ESCS/FEPECS/SES-DF;

7 Psicóloga residente em Saúde Mental Infantojuvenil da Escola Superior de Ciências da Saúde ESCS/FEPECS/SES-DF;
} 


\begin{abstract}
This descriptive study seeks to report the experience of residents of the Child Mental Health Residency Program in a Child Psychosocial Care Center (CAPSi DF) in the context of a pandemic related to the new coronavirus (COVID -19). Considering the guidelines of the World Health Organization, Ministry of Health and the contingency plans of the local government, the psychosocial rehabilitation actions in children's mental health have undergone adaptations. A qualitative methodology based on psychosocial care, CAPS guiding principles, psychiatric reform and literature on madness, culture and society was used to promote a critical reflection on the necessary task of sustaining the logic of psychosocial care in times of social isolation and worsening risks to the mental health of children and adolescents.
\end{abstract}

Key words: Pandemic; Psychosocial Care; Madness; Child and Adolescent Mental Health; Public health;

\title{
Introdução
}

No mês de dezembro de 2019, na cidade de Wuhan, província de Hubei, na China, surgiu um novo coronavírus (severe acute respiratory syndrome coronavirus 2 ou SARS-CoV2) que foi identificado como agente etiológico de um surto de pneumonias ${ }^{1,2,3,4}$. A enfermidade passou a ser designada como COVID-19 (coronavírus disease 2019) foi classificada como pandemia, por sua veloz dispersão com transmissão de pessoa a pessoa, pela sua internacionalização, atingindo outros continentes, e pelos novos surtos relacionados à transmissão comunitária $1,2,3,4$

A Organização Mundial de Saúde (OMS) define pandemia como uma disseminação mundial de uma nova patologia. Foi diante do aumento do número de casos, óbitos e países afetados, que em 30 de janeiro de 2020, a OMS declarou que o episódio constituía uma Emergência de Saúde Pública de Importância Internacional (ESPII). ${ }^{1}$ Assim, em 11 de março de 2020, com a notificação de mais de 110 mil casos e 4 mil óbitos em países de todos os continentes, a OMS declarou a pandemia de COVID-19, e posteriormente, o Brasil, em 3 de fevereiro de 2020 também declara a epidemia como de Emergência em Saúde Pública de Importância Nacional (ESPIN). ${ }^{2,3}$ 
Atualmente, nota-se um esforço cada vez mais intenso por parte das organizações de saúde e do poder público no sentido de conter o avanço e disseminação do vírus, que é caracterizado por alta capacidade de transmissão e indução de quadros de infecção respiratória severa, além de ausência de imunidade prévia na população humana e a inexistência de vacina contra este vírus, acarretando no crescimento de casos em caráter exponencial. ${ }^{4}$ Nesse contexto, são indicadas intervenções não farmacológicas (INF), visando inibir a transmissão entre humanos, desacelerar o espalhamento da doença, e consequentemente diminuir e postergar o pico de ocorrência na curva epidêmica, consequentemente, é possível reduzir a demanda instantânea por cuidados de saúde e mitigar as consequências da doença sobre a saúde das populações, incluindo a minimização da morbidade e da mortalidade associadas. ${ }^{4,5,6,7}$

Nesse contexto, é fundamental a atuação do Sistema Único de Saúde (SUS) e das demais áreas do sistema de proteção social de forma articulada, de modo a se favorecer a adesão das pessoas às INF e minimizar os impactos deletérios das medidas comunitárias. A proteção da saúde pública deverá ser norteadora das decisões a serem tomadas pelos gestores. É fundamental que essas decisões sejam baseadas nas melhores evidências disponíveis e comunicadas de forma transparente, para se promover a confiança da população. As orientações das autoridades e a adesão das pessoas às INF serão determinantes para o curso da epidemia da COVID-19 no Brasil. . $^{8,9,10}$

No Brasil, em 6 de fevereiro de 2020, foi sancionada a Lei no 13.979, que dispõe sobre as medidas para enfrentamento da epidemia da COVID-19 e elenca as INF comunitárias que podem ser adotadas. Assim, conforme orientações de organismos internacionais (OMS) e Ministério da Saúde (MS) os governos locais, em consonância com tais instituições, aplicaram Planos de Contingenciamento das atividades públicas e privadas para que não exista uma maior proliferação do COVID 19, o que impacta diretamente as ações da atenção psicossocial. ${ }^{8,9,10}$

Diante dessa breve introdução sobre os impactos do coronavírus no Brasil e as medidas internacionais, nacionais e locais recomendadas, o presente estudo visa contribuir e promover uma reflexão crítica sobre à necessidade de sustentação da lógica da atenção psicossocial em contexto de pandemia. Tendo em vista, o agravamento do sofrimento de ordem objetivo e subjetiva que afeta à população, entre elas à infantojuvenil, no que tange o estresse, o medo, ausência de atividades, o isolamento social, à violência doméstica, desemprego, fome etc.

\section{Loucura, cultura e sociedade: Breve histórico da Atenção Psicossocial Infantojuvenil}


Segundo definição do dicionário Aurélio", a loucura trata-se de "Estado ou condição de louco; insanidade mental; Falta de discernimento; irreflexão, absurdo, insensatez, doidice, louquice" ou ainda "Tudo que foge às normas, que é fora do comum". Pelbart (1990 p. 133) 12, traz a distinção entre os conceitos de louco e loucura. Para ele, louco refere-se a um "personagem social discriminado, excluído e recluso", enquanto a loucura (chamado por ele de desrazão) seria caracterizada pela "estranheza, ameaça, alteridade radical, tudo aquilo que uma civilização enxerga como o seu limite, o seu contrário, o seu outro, o seu além".

A "loucura" como fenômeno aparece sendo relatada de maneira inicial na antiguidade grega e romana. ${ }^{13} \mathrm{O}$ louco nesse período tem sua existência e presença consideradas essenciais, por ser interpretado como detentor das manifestações sobrenaturais motivadas por deuses. Posteriormente a isso, vê-se na história que a loucura deixa de ocupar esse lugar de admiração e essencialidade, passando, então, a ocupar lugares relacionados à exclusão e perseguição.

Tanto na Idade Média, quanto no período Renascentista, século XVII ou na Era Clássica, o que se apreende é que apesar das diferenças de como a exclusão do louco era realizada - seja por meio de discursos religiosos, higienistas ou médicos - tem-se em comum, o fato de que a história da loucura está diretamente ligada ao seu afastamento do seio social. Assim como acontecia com todos aqueles que não se encaixavam na normalidade desejada. Entende-se que houve sempre o mecanismo de tentativa de libertar a sociedade da loucura, excluindo qualquer possibilidade de respeito e valorização da singularidade daquele em sofrimento mental. $\mathrm{O}$ fato ocorrido no período Renascentista que ficou conhecido como Nau dos Loucos, retrata bem todas as demais eras: "Essas embarcações dispunham de um valor simbólico, como um ritual que libertava a sociedade dos doidos" (p.4). ${ }^{13}$

O modelo asilar começou a ser repensado logo após a Segunda Guerra Mundial, a partir da mobilização da sociedade a favor dos direitos humanos. Essa movimentação levou a difusão da Reforma Psiquiátrica em diversos países, com o objetivo principal de implementar uma política de saúde mental inclusiva, equânime, extra-hospitalar e com bases comunitárias. ${ }^{14}$ No Brasil não foi diferente, o mesmo cenário sociopolítico da época impulsionou as mudanças na assistência em saúde mental, sendo em 1980 traçadas as primeiras mudanças a serem efetivadas nessa área, tendo em vista a criação de uma rede de serviço que seriam extra-hospitalares como um dos dispositivos da Reforma. ${ }^{15}$

Para Costa - Rosa (2013, p.12) ${ }^{16}$ a Reforma Psiquiátrica (RP) é um movimento mundial de luta por transformações nas práticas de Atenção ao sofrimento psíquico e mental, atua como 
um movimento ético-político com o teor de contestação que deixa expresso, de modo iniludível, seu caráter de alteridade à psiquiatria. Essa Atenção visa a construção de novas metodologias, processos de trabalho, visibilidade nas práticas de cuidado ao sofrimento psíquico contrárias às práticas asilares.

No modelo da Atenção Psicossocial, radicalmente oposto ao modelo AsilarHospitalocêntrico hegemonizado pelas práticas biomédicas, ressaltam-se: a leitura biopsicossocial do sujeito; a mobilização e participação do sujeito no tratamento de forma indispensável; a pertinência do indivíduo em um grupo familiar e social, sendo o indivíduo e sua família multiplicadores de mudanças; a participação da família devem superar o modelo assistencial e passivo, apostando em uma atuação mais ativa cooperativa.

Os primeiros sinais de possibilidade de transformação da assistência psiquiátrica no Brasil tiveram início apenas no final da década de 1970. Segundo Vasconcelos (2002, p. 49$50)^{17}$ era necessária "uma revisão dos paradigmas, que reduziram e aprisionaram a loucura como objeto de um saber exclusivamente médico, e a superação das formas assistenciais segregadoras, dentro do sistema público de saúde, que desrespeitam a cidadania do louco”. Em referência às políticas de atenção à pessoa (adulto e/ou criança e adolescente) com transtorno mental ou sofrimento psíquico, as principais mudanças geradas pela implantação dos novos serviços de atenção à saúde mental, com o movimento da Reforma Psiquiátrica, estão relacionadas com a cidadania dos usuários, a valorização da inserção dos familiares no tratamento e o processo de desmistificação da loucura junto à comunidade.

Apesar do Movimento da Reforma Psiquiátrica avançar em uma proposta de cuidado ampliado em relação à pessoa em sofrimento psíquico e reorientar o lugar da loucura na intervenção em saúde pública, cabe ressaltar e sublinhar a invisibilidade das crianças e dos adolescentes nesse debate. Historicamente, o sujeito-objeto em destaque de tais políticas foi a pessoa adulta em sofrimento mental, inviabilizando e inserindo de forma tardia no referido debate a infância, ficando assim desassistidas ou submetidas a processos de institucionalização externos ao sistema psiquiátrico asilar, que, por décadas, foram invisíveis até mesmo ao vigoroso movimento da Reforma Psiquiátrica brasileira. ${ }^{18}$

Couto (2015 p.19) ${ }^{19}$ menciona que os caminhos para o desenvolvimento de uma Política de Saúde Mental para Crianças e Adolescentes (SMCA) no Brasil só foram propostos no início do século XXI. Antes desse momento, o país não dispunha de formulações pelo setor da saúde mental que orientassem a construção de uma rede de cuidados para crianças e adolescentes com problemas mentais, ficando as ações assistenciais, quando existentes, circunscritas à 
agenda dos setores da assistência social e educação, com propostas mais reparadoras e disciplinares do que propriamente clínicas ou psicossociais. Ou seja, o conceito de criança surge assentado sobre a lógica higienista, onde a criança ideal é destinada a transformar-se no indivíduo racional, centrado em sua consciência, em um "sujeito suposto-adulto". ${ }^{20} 21$

França (2017 p. 70) ${ }^{20}$ destaca que no Brasil um importante destaque no campo dos direitos Infantojuvenil foi o Estatuto da Criança e do Adolescente (ECA) Lei nº869/1990 atrelada à Constituição Federal de 1988, conhecida como constituição cidadã. Tal contexto, amplia o local social, moral e jurídico das crianças e adolescente, compreendo-os como sujeitos de direitos, entre eles, a saúde. Mesmo diante de tantos avanços, no que concerne à saúde mental-Infantojuvenil, seus efeitos só serão refletidos na III Conferência Nacional de Saúde Mental (2001) ano que foi promulgada a Lei 10.216 e que foi um marco para a saúde mental de crianças e adolescentes. ${ }^{19}$

As políticas de saúde mental (SM) e atenção psicossocial (AP) no SUS têm relação direta com a ideia-proposta-projeto-movimento-processo, diante das problemáticas apresentadas no cenário de violação de direitos. Surgiu o primeiro movimento em 1970 de profissionais recém formados que ampliaram o olhar a respeito das práticas desenvolvidas e invisibilidade do sofrimento. A partir dessa mobilização irá se constituir a redemocratização das políticas públicas, vale salientar que este é o primeiro movimento dos trabalhadores da saúde mental (MTSM) com o objetivo de ressignificar a atuação na assistência psiquiátrica e após organizarem eventos como associação de usuários e familiares , congressos nacionais, fóruns de direitos humanos e saúde mental.

Diante da atuação exitosa que rompe com os estigmas cristalizados no que se refere ao sofrimento mental, o coletivo de profissionais se torna um movimento social, não apenas com os próprios "loucos" e seus familiares, mas também com outros ativistas de direitos humanos. A partir de então se transforma em Movimento da Luta Antimanicomial (MLA), o coletivo passou a organizar ações por todo o país. Elemento decisivo para esta ampliação foi a criação, já em Bauru, do Dia Nacional da Luta Antimanicomial. O dia 18 de Maio serviria para despertar o pensamento crítico na sociedade sobre a violência institucional da psiquiatria e a exclusão das pessoas em sofrimento psíquico. Pode-se considerar que o objetivo teve êxito na medida em que, desde então, são realizadas atividades políticas, científicas, culturais e sociais não apenas no dia em questão, mas por todo o mês de maio, que passou a ser considerado o Mês da Luta Antimanicomial. ${ }^{22}$ 
Os conceitos de institucionalismo, poder institucionalizante e instituição total predominavam nos discursos de então. É no final dos anos 1980 que surge a perspectiva de criar serviços que deem início a práticas inovadoras. Em São Paulo foi criado o primeiro Centro de Atenção Psicossocial (CAPS) e outras iniciativas começaram a surgir. Outros marcos importantes das políticas de saúde mental no SUS foram estabelecidos pela Portaria/GM no 336, de 19/02 de 2002, que redefiniu os CAPS em relação à sua organização, ao porte, à especificidade da clientela atendida. Passaram a existir CAPS I, CAPS II, CAPS III, CAPSi (infantil ou infanto-juvenil) e CAPSad (álcool e drogas). ${ }^{22}$

\section{CAPSi}

O Centro de Atenção Psicossocial Infantojuvenil (CAPSi), é um dispositivo de saúde mental que opera no eixo do sujeito, da família, da sociedade e da cultura, buscando atravessar, significar e renovar os contratos sociais. Constitui-se como um dispositivo aberto de assistência diária para crianças e adolescentes com transtornos mentais graves - “autismo, neuroses graves, psicoses, ou todo aquele que devido a sua condição psíquica está impossibilitado de estabelecer laços sociais, utilizar seus recursos pessoais e lidar com as demandas do dia a dia" (p.14) $)^{23}$ - e/ ou dependentes de álcool ou outras drogas. Os adolescentes com idade a partir de 16 anos em uso abusivo ou dependência de substâncias psicoativas passam a ser atendidos no Centro de Atenção Psicossocial de Álcool e outras drogas (CAPS AD), adolescentes em cumprimento de medida socioeducativa, crianças e adolescentes em situação de rua. Sua especificidade está na necessária adaptação que deve ser feita às faixas etárias que o serviço compreende - crianças e adolescentes até os 18 anos.

Assim como os demais Centros de Atenção Psicossocial, criados a partir da necessidade de desinstitucionalização dos usuários do serviço de saúde mental, o CAPSi deve ser substitutivo às internações em hospitais psiquiátricos. ${ }^{24}$ Esse fato, se deve principalmente à aprovação da Lei No 10.216/01 da Reforma Psiquiátrica, a Portaria 336/02, e assim definido em 2001 pelo Ministério da Saúde.

O CAPSi da Asa Norte - cenário de pesquisa do presente trabalho - tem como área de abrangência as regiões Central, Centro-Sul, Sudoeste e Leste de Brasília, possuindo em média 180 usuários ativos, no que tange o desenvolvimento das ações de cuidado aos usuários e familiares realizadas por esse serviço, a atuação ocorre em equipe multiprofissional por meio 
dos atendimentos individuais, atendimentos coletivos, atendimento familiar, assembleia de usuários e pais, promoção de contratualidade no território, ação de atenção psicossocial, articulação intersetorial, intervenção no domicílio, matriciamento das unidades básicas de saúde, atenção a crise, fortalecimento do protagonismo do usuário, práticas expressivas e comunicativas, práticas integrativas. O acompanhamento terapêutico ocorre no espaço físico e no território propiciando promoção de saúde na rua com o envolvimento dos atores da comunidade com direcionamento para o olhar integral do sujeito, conforme " conversações e pensamentos que ocorrem durante um passeio, caminhando - peripatetismos - são uma ferramenta para entender uma série de experiências clínicas realizadas fora do consultório, em movimento"(LANCETTI, 2006, p. 19) ${ }^{25}$

\section{Desafios de sustentar a lógica da Atenção Psicossocial Infantojuvenil e seus princípios}

Diante da explanação realizada, percebe-se que a atual lógica da atenção psicossocial é contrária ao isolamento dos usuários e da loucura. Além disso, existe um esforço para que não existam retrocessos referentes à institucionalização e a consequente exclusão do indivíduo em sofrimento mental - como já ocorrido anteriormente ao retomarmos a história da evolução do "tratamento" da loucura. Porém, ao nos encontrarmos em um momento delicado de pandemia e crise mundial ocasionado pela COVID-19, em que a proteção dos sujeitos está diretamente relacionada ao isolamento, vemo-nos com enormes desafios a serem enfrentados e adaptações a serem realizadas. É necessário refletirmos acerca dos princípios dos CAPS para que o funcionamento desse serviço - que presta uma atenção humanizada - continue exercendo o seu papel de resistência no cuidado com as pessoas em sofrimento mental.

Frente às políticas e aos ideais que garantem o cuidado em saúde mental Infantojuvenil em liberdade, se faz necessário pensar qual a realidade em que o CAPSi Asa Norte se encontra e quais são os desafios para que se sustente essa conquista histórica em meio a Pandemia do COVID-19. À vista disso, alguns princípios e ideais orientam o cuidado em Saúde Mental e assim, incluindo a Saúde Mental Infantojuvenil, sendo esses: Crianças e adolescentes enquanto sujeitos responsáveis por demanda e sintoma; Acolhimento universal; Encaminhamento implicado e corresponsável; Construção permanente da rede e da intersetorialidade; Trabalho no território; Avaliação das demandas e construção compartilhada das necessidades de saúde mental. ${ }^{26}$

Assim sendo, é imprescindível a contextualização histórica do CAPSi Asa Norte, o qual durante 15 anos era o único CAPSi que atendia o DF e o entorno, localizado no Plano Piloto e 
sofrendo diretamente com a lógica de setorialização de Brasília, uma vez que ainda se encontra dentro de um ambulatório de saúde mental Infantojuvenil e com dificuldades de ser remanejado para um lugar próprio. Dessarte, mesmo com a criação de novos CAPSi em Brasília, destacase que o CAPSi Asa Norte recebe uma demanda das mais diversas regiões de Brasília/DF, distantes uma das outras e com realidades sócio, históricas e culturais diferentes, com um grande desafio já posto anterior a Pandemia: Como um serviço territorializado lida com territórios estruturais completamente diferentes e sem estar no território fisicamente? Essa indagação traz muitas reflexões, entretanto podemos afirmar que a articulação de estar em cada território era e continua sendo uma luta diária e constante do serviço.

Essa contrariedade posta, expõe uma angústia ainda maior, pois com a Pandemia a tentativa de estar nos mais diversos territórios fisicamente e realizar as articulações intersetoriais se torna impedida e assim um grande "ouro" da Atenção Psicossocial é obstaculizado, o trabalho territorial. Nesse aspecto, Faleiros $(2011)^{27}$ ressalta que a construção das redes é processual e dinâmica envolvendo tanto a família como os amigos, os vizinhos, a comunidade, assim como as redes formais das organizações de saúde, assistência, educação entre outras, a partir dos sujeitos implicados. Sendo assim, as redes primárias são formadas pelas famílias e as redes secundárias pelas organizações sociais, de modo a articular a outras redes para fortalecer os sujeitos.

Em relação a idas ao território, algo muito frequente do CAPSi, está sendo evitado por conta do COVID-19, entretanto o CAPSi continua com sua forte potência de articulação de rede por mecanismos que não necessitem a aproximação física, se comunicando com os agentes do território formal por meios oficiais disponibilizados pelo GDF e produzindo sua intensa articulação territorial. Porém, como o CAPSi Asa Norte sustentará sua lógica de cuidado com os 180 usuários ativos, bem como os casos que têm acessado o serviço para inserção em acolhimento e posteriormente acompanhamento terapêutico.

Atualmente o CAPSi Asa Norte tem como um dos desafios a falta de condições de trabalho dentre elas: falta de equipamento público e escassez do recurso humano, além disso, com os afastamentos dos profissionais em grupo de risco e por licença médica se encontra ainda mais árduo o trabalho. Nesse aspecto, os profissionais que não se encontram afastados do trabalho, diariamente se desdobram para que os atendimentos continuem, das mais variadas formas, contando com a presença da Residência Multiprofissional em Saúde Mental Infantojuvenil, o qual se torna potência e motor de sustentação da lógica da Atenção Psicossocial. 
Diante do "corpo orgânico" estar em perigo, aglomerações de pessoas devem ser evitadas, assim grupos e coletivos são necessariamente interrompidos, pois como pontua Mezzina, 2020 "os locais de encontro e troca, hoje podem se transformar em locais de infecção". ${ }^{28}$ Os coletivos, principais estratégias de escuta e cuidado do CAPSi Asa Norte, foram suspensos e a demanda de 180 usuários e seus familiares emergem de forma individual. Nesse momento, temos um grande aliado: os teleatendimentos. Essa modalidade de escuta, afeta o princípio pontuado acima de "Crianças e adolescentes enquanto sujeitos responsáveis por demanda e sintoma", pois muitas vezes não é possível escutá-los, se restringindo somente a voz de responsáveis legais. Nesse sentido, os CAPSi's devem buscar alternativas de dar voz aos protagonistas de seu cuidado, buscando sempre endereçar seu lugar de fala e alternativas para que as angústias de crianças e adolescentes sejam aliviadas enquanto protagonistas.

A participação social é fundamental na construção dessas políticas e redes de atenção, uma vez que, a essência do movimento é a luta por direitos e afirmação da cidadania, que produz novos sujeitos de direitos. Sendo assim, é fundamental que esses espaços de mobilização sejam cada vez mais ocupados pela população, entendendo que esse movimento político pela saúde mental siga de acordo com os preceitos instituídos pela reforma psiquiátrica. $^{29}$

No princípio do acolhimento universal, localiza-se em meio à pandemia a necessidade ainda maior desse ideal. Nesse momento, ter conhecimento de como a rede está funcionando e os canais de comunicação, se fazem extremamente necessário, para que uma escuta alivie a angústia, um encaminhamento implicado seja resolutivo e uma maior possibilidade de articulação da rede e intersetorialidade ocorra de forma assertiva.

Por ser um serviço que atua com os sofrimentos psíquicos graves, os CAPSi lidam diretamente com a crise do sujeito e nesse momento com as demandas apresentadas pela crise mundial e seus desafios. Nesse sentido, políticas de saúde mental devem ser elaboradas, pois após o corpo orgânico estar ameaçado, o corpo psíquico da sociedade se encontrará desalentado e não somente para aqueles que um dia foram diagnosticados com algum sofrimento mental. $\mathrm{O}$ "corpo social", pontuado por Basaglia frequentemente não se encontra em meio ao COVID$19 .{ }^{28}$

O bloqueio, o estar fechado em casa, que a Itália e agora outros países estão experimentando e vivendo, é um enorme experimento coletivo, uma nova Norma: uma condição generalizada, que afeta todos, especialmente aqueles que estão realmente em casa e não devem ir todos os dias aos seus locais de trabalho, assistência médica, produção ou serviços essenciais. ${ }^{28}$ 
Com os teleatendimentos e a diminuição de fluxo de pessoas no serviço, deve-se prezar por orientações e encaminhamentos implicados. O monitoramento telefônico (teletrabalho) deve ser intenso e afetivo preconizando assim o acesso aos direitos sociais básicos e demais políticas públicas.

Por fim, como pontuado na história da loucura e reforma psiquiátrica, o isolamento social de grupos que divergiam de uma normatização da sociedade, como os sujeitos em sofrimento psíquico, era naturalizado e somente com uma luta histórica desvelou-se os reais sentidos dessa prática, invertendo, com a lógica da Atenção Psicossocial, o cuidado em Saúde Mental. Entretanto, atualmente, o isolamento é permitido e imposto a todos, distanciando a proximidade da lógica da Atenção psicossocial. Os serviços que destoam da lógica de exclusão são os que mais sofrem, aqueles nascidos com a Reforma Psiquiátrica. Dessa forma, eminentemente deve-se elaborar significado para a quarentena e o isolamento, para além de regras de higiene. ${ }^{28}$

\section{Relato de estratégias CAPSi Asa Norte- DF}

$\mathrm{Na}$ conjuntura que se configura o estado emergencial de saúde pelo COVID-19, observa-se que o SUS experimenta diariamente caráter mutatório correspondente ao enfrentamento a pandemia. Não distante, o CAPSi passa por desafios enquanto sustentação de um serviço de saúde mental Infantojuvenil. O CAPSi Asa Norte, cenário de saúde pública do Distrito Federal, em meio à pandemia pelo COVID-19, voltou-se para elaboração de adaptações quanto a configuração do serviço visando a manutenção do atendimento ao público, ao tempo que órgãos como Ministério da Saúde e Governo do Distrito Federal lançavam instrumentos norteadores para a construção do plano de contingenciamento dos serviços de saúde, com abrangência para todos os níveis de atenção à saúde.

Por sua vez, as repercussões das indicações de medidas de segurança como distanciamento social; permanecer, preferencialmente, em casa; vigilância quanto a higiene do local entre outras ${ }^{30}$ indicam agravamento no que tange a saúde mental populacional como referido na cartilha "Saúde Mental e Atenção Psicossocial na Pandemia COVID-19" direcionada aos gestores dos serviços de saúde mental:

Ainda que a maior parte dos problemas psicossociais sejam considerados reações e sintomas normais para uma situação anormal, estima-se um aumento da incidência de transtornos psíquicos (entre um terço e metade da população) de acordo com a magnitude do evento, o grau de vulnerabilidade psicossocial, o tempo e a qualidade das ações psicossociais na primeira fase da resposta à epidemia. ${ }^{3 l}$ 
Diante do exposto, o CAPSi Asa Norte visou cumprir as diretrizes de segurança ao passo que há a compreensão da importância do funcionamento da unidade no contexto de pandemia para atender as demandas de saúde mental Infantojuvenil. Para tal, a estratégia inicial do CAPSi Asa Norte implicou na redução do fluxo de pessoas dentro do serviço visando a diminuição da exposição ao risco de contaminação por COVID-19. Essa abordagem se deu após identificação da fragilidade devido a possível aglomeração de pessoas, não apenas dentro do espaço físico da unidade, como também no trajeto percorrido para acessar o CAPSi. Sendo assim, houve a suspensão dos coletivos (entende-se coletivo como dispositivo terapêutico ativo produtor de singularidades ${ }^{32}$ ) e a elaboração da nova configuração de funcionamento.

Os pacientes foram classificados em intensivos, semi intensivos e não intensivos como instrumento de identificação da gravidade dos casos visando aplicar intervenções compatíveis com as demandas particulares. O corpo técnico do CAPSi se organizou para que as equipes de referência de cada coletivo mantivessem suas intervenções direcionadas aos grupos de pacientes já vinculados, em respeito ao vínculo terapêutico construído previamente e assim, dando seguimento ao processo terapêutico dessas crianças e adolescentes. Em suma, o direcionamento é para os mediadores dos coletivos permanecerem em contato com os integrantes, no entanto, o atendimento nesse contexto de pandemia ocorre de forma individual e de acordo com as demandas apresentadas pelo usuário e seus familiares.

Sendo assim, os pacientes classificados como intensivos tem acompanhamento por teleatendimento uma ou mais vezes por semana e, quando necessário - crises, situação de vulnerabilidade, agravo em sofrimento psíquico - atendimento individual in loco, enquanto os pacientes semi intensivos e não intensivos são acompanhados por teleatendimento com maior intervalo de tempo entre as ligações e nas situações em que ocorra a necessidade da articulação intersetorial tem sido mantida a aproximação dos órgãos públicos com as devidas orientações e encaminhamentos implicados.

A partir da necessidade de reformulação da estratégia de atendimento aos usuários e após os conselhos regionais de múltiplas categorias de saúde liberaram para que os profissionais pudessem realizar atendimento a distância utilizando tecnologia em prol da assistência. Dessarte, o teleatendimento surge no serviço como recurso no contexto da pandemia por COVID-19 em vista da restrição de contato presencial na saúde mental Infantojuvenil. Com a inserção dessa nova forma de assistência, a equipe multiprofissional utilizou o espaço da reunião em equipe em um de seus instrumentos técnicos operativos a ação 
de atenção psicossocial para que o serviço unificasse a compreensão e utilização tecnológica na clínica de saúde mental.

Além de mudanças organizacionais, observa-se aumento da fragilidade social em grupos já expostos a vulnerabilidade social, com isso, entende-se o papel do CAPSi na atuação territorial por meio do fortalecimento da Rede de Atenção Psicossocial (RAPS) para contemplar cuidado holístico e atendimento de demandas emergentes que transcendem a capacidade do serviço de atenção à saúde mental Infantojuvenil. São integrantes da RAPS: "Centros de Atenção Psicossocial (CAPS); os Serviços Residenciais Terapêticos (SRT); os Centros de Convivência e Cultura, as Unidade de Acolhimento (UAs), e os leitos de atenção integral (em Hospitais Gerais, nos CAPS III)". ${ }^{33}$

Para além das intervenções visando atender os casos de abrangência do serviço, a gestão produziu um documento enviado a Diretora Regional de Atenção Secundária à Saúde (DIRASE) disponibilizando a equipe para acolher, por meio de teleatendimento, às demandas de sofrimento psíquico que permeiam tanto as pessoas que testaram positivo para o COVID19 quanto para seus familiares entendendo a fragilidade desencadeada pela configuração da pandemia.

Posto isso, as formas de enfrentamentos aplicadas pelo CAPSi Asa Norte foram reduzir o fluxo de pessoas circulando no serviço; contemplar por teleatendimento todos os pacientes ativos e receber in loco crises e os casos intensivos mantendo os protocolos de segurança ${ }^{28}$ além de oferecer máscaras cirúrgicas e máscaras faciais de acetato para os funcionários; fortalecimento da RAPS como extensão das intervenções; capacitação da equipe para atuar no contexto de pandemia por meio de debates e promoção da educação permanente nas reuniões de equipe.

\section{Considerações finais}

Ao analisarmos o contexto de pandemia da COVID-19 em que nos encontramos e relacioná-lo com as dificuldades perante a atuação nos serviços de saúde mental - a destacar o CAPSi - nos vemos diante de um desafio a ser enfrentado em defesa da atual e importante lógica da atenção psicossocial. Defrontamo-nos com um dilema que de um lado implica-nos o reconhecimento da clínica da saúde mental que se esforça pela prestação de serviços com o objetivo de promover mudanças através do encontro qualificado entre os sujeitos que cuidam e os sujeitos que necessitam de cuidados e de outro lado com a necessidade de cumprir-se a 
medida comunitária de afastamento como forma de proteger a população de um risco eminente de contágio do coronavírus.

Em especial, ressaltamos a empreitada do CAPSi Asa Norte - DF na humanização da atenção à saúde mental e sua importância na concretização dos ideais defendidos pela reforma psiquiátrica. Diante da atual crise pandêmica e das diversas limitações existentes, o serviço desenvolveu estratégias para que mesmo com a suspensão dos atendimentos coletivos, o usuário e suas famílias permaneçam assistidos frente a suas demandas de cuidado.

Destaca-se que o presente trabalho, não pretende encerrar as discussões a respeito da temática de como se dará a atuação dos serviços de saúde mental em meio a pandemias como a COVID-19, mas servir como base para reflexões sobre como estratégias foram construídas pela equipe do CAPSi Asa Norte - DF e assim, galgar-se novos desafios e projetos. Por fim, o desenvolvimento do trabalho necessita do compromisso coletivo em articulação com a rede intersetorial como mecanismo de proteção e cuidado integral visando o reconhecimento do sujeito em sua totalidade.

\section{Referências Bibliográficas}

1. National Health Commission of the People's Republic of China. New coronavirus cases rise to 571 in Chinese mainland [Internet]. China: 2020 [cited 2020 Mar 16]. Available from: http://en.nhc.gov.cn/2020-01/23/c_76004.htm

2. World Health Organization (WHO). Novel Coronavirus - Republic of Korea (exChina) [Internet]. Geneva: WHO; 2020 [cited 2020 Mar 16]. Available from: https://www.who.int/csr/don/21-january-2020-novel-coronavirus-republic-ofkorea-exchina/en/

3. European Centre for Disease Prevention and Control [ECDC]. Geographical distribution of 2019-nCov cases. Situation update worldwide, as of 27 March 2020 [Internet]. ECDC; 2020 [cited 2020 Mar 16]. Available from: https:// www.ecdc.europa.eu/en/geographicaldistribution-2019-ncov-cases

4. Anderson RM, Heesterbeek H, Hollingsworth TD. How will country-based mitigation measures influence the course of the COVID-19 epidemic? Lancet [Internet]. 2020 Mar [cited 2020 Mar 27];395(10228):931-4. Available from: https://doi.org/10.1016/S0140$6736(20) 30567-5$ 
5. Centers for Disease Control and Prevention [CDC]. Coronavirus Disease 2019 (COVID-19). Cases in U.S [Internet]. USA: CDC; 2020 [cited 2020 Mar 16]. Available from: https://www.cdc.gov/coronavirus/2019-ncov/cases-in-us.html

6. Qualls N, Levitt A, Kanade N, Wright-Jegede N, Dopson S, Biggerstaff M, et al. Community mitigation guidelines to prevent pandemic influenza - United States, 2017MMWR Recomm Rep [Internet]. 2017 Apr [cited 2020 Mar 27];66(1):1-32. Available from: https://doi.org/10.15585/mmwr.rr6601a1

7. Ferreira MJ, Irigoyen MC, Consolim-Colombo F, Saraiva JFK, Angelis KD. Vida Fisicamente Ativa como Medida de Enfrentamento ao COVID-19. Sociedade brasileira de cardiologia. 2020. https://doi.org/10.36660/abc.20200235.

8. World Health Organization. Statement on the second meeting of the International Health Regulations (2005) Emergency Committee regarding the outbreak of novel coronavirus (2019nCoV) [Internet]. Geneva: World Health Organization; 2020 [cited 2020 Mar 27]. Available from: $\quad$ https://www.who.int/news-room/detail/30-01-2020-statement-on-the-secondmeetingof-the-international-health-regulations-(2005)-emergency-committee-regarding-theoutbreak-of-novel -coronavirus-(2019-ncov)

9. Croda JHR, Garcia LP. Resposta imediata da Vigilância em Saúde à epidemia da COVID19. Epidemiol Serv Saúde [Internet]. 2020 [citado 2020 Mar 26];29(1):e2020002. Disponível em: https://doi.org/10.5123/s1679-49742020000100021

10. Garcia LP, Duarte E. Intervenções não farmacológicas para o enfrentamento à epidemia da COVID-19 no Brasil. Epidemiol. Serv. Saude. 2020.

11. Aurélio. Dicionário da língua portuguesa. Curitiba: Ed. Positivo, 2009.

12. Pelbart PP. Manicômio Mental: a outra face da clausura. In Lancetti A. Saúde Loucura. São Paulo: Hucitec; 1990.

13. Millani HdFB, Valente MLLdC. O Caminho da Loucura e a Transformação da Assistência aos Portadores de Sofrimento Mental. Revista Eletrônica Saúde Mental Álcool Drogas. 2008.

14. Paiano M, Maftum MA, Haddad MdCL, Marcon SS. Ambulatórios de Saúde Mental: Fragilidades apontadas por profissionais. Texto contexto Enferm. 2016; 25(3). 
15. Santos YFd, Oliveira IMFFd, Yamamoto OH. O ambulatório da saúde mental no contexto da Reforma Psiquiátrica em Natal RN. Psicologia Argumento. 2009 dezembro; 27(59).

16. Costa-Rosa Ad. Atenção Psicossocial além da Reforma Psiquiátrica: contribuições a uma Clínica Crítica dos processos de subjetivação na Saúde Coletiva. 2013th ed. São Paulo: Unesp; 2013.

17. Vasconcelos EM. Saúde mental e serviço social: o desafio da subjetividade e da interdisciplinaridade. 2nd ed. São Paulo: Cortez; 2002.

18. Couto MCV. Por uma Política de Saúde Mental para crianças e adolescentes. In Ferreira T. A criança e a saúde mental:enlaces entre a clínica e a política. Belo Horizonte: Autêntica; 2004. p. 61-74.

19. Couto MCV, Delgado PGG. Crianças e adolescentes na agenda política da saúde mental brasileira: inclusão tardia, desafios atuais. Psic. Clin. 2015; 27(1).

20. França VNd. Da clínica à gestão: uma política para os CAPSi do DF. Tese de Doutorado. 2017.Disponível em: https://repositorio.unb.br/handle/10482/31507.

21.Bercherie P. A clínica psiquiátrica da criança. In Cirino O. Psicanálise e psiquiatria com crianças: desenvolvimento ou estrutura. Belo Horizonte: Autêntica; 2001. p. 129-144.

22. Amarante $\mathrm{P}$, Nunes MdO. A reforma psiquiátrica no SUS e a luta por uma sociedade sem manicômios. Ciência \& Saúde Coletiva. 23(6), 2018.

23. Vieira ACMD, Pinto JM. Saúde Mental Infantojuvenil e o Papel do CAPS: Uma Análise a Partirde Oficinas Com Profissionais de Franco da Rocha (SP). Monografia. 2018

24. Ministério da Saúde. O SUS de A Z: garantindo saúde nos municípios. Brasília: DF, 2009a.

25. Lancetti A. Clínica peripatética São Paulo: Hucitec; 2006.

26. Brasil. Ministério da Saúde. Caminhos para uma Política de Saúde Mental Infantojuvenil. Brasília, 2005.

27.Faleiros VdP. Estratégias em Serviço Social. 10th ed. São Paulo: Cortez; 2011. 
28. Mezzina R. Saúde mental: serviços, indivíduos e o corpo social na época do coronavírus. Trieste - Itália. Marco. 2020.

29. Braga CP, D'Oliveira AFPL. Políticas públicas na atenção à saúde mental de crianças e adolescentes: percurso histórico e caminhos de participação. Ciên. saúde coletiva [online]. 2019; 24(2). https://doi.org/10.1590/1413-81232018242.30582016.

30. Ministério da Saúde (BR). COVID-19: Como se proteger. Brasil, 2020. Disponível em: $<$ https://coronavirus.saude.gov.br/sobre-a-doenca\#como-se-proteger>

31. Fiocruz. Saúde Mental e Atenção Psicossocial na Pandemia COVID-19: Recomendações para gestores. Pesquisadores do Centro de Estudos e Pesquisas em Emergências e Desastres em Saúde; 2020.

32. Oury J. O Coletivo São Paulo: Hucitec; 2009.

33. Ministério da Saúde (BR). O que é a Rede de Atenção Psicossocial. Brasil, 2013. Disponível em: $<$ https://bvsms.saude.gov.br/bvs/folder/conheca_raps_rede_atencao_psicossocial.pdf $>$ 